\title{
REGISTER DER ERWÄHNTEN UND BEARBEITETEN TEXTE
}

Wichtige Passagen zu Inhalt, Datierung und Interpretation sind fett gedruckt.

Abregié de Troyes 107, 144, 151, 335, 360

Adrianus de Budt: Chronik der Religiosen der Abtei Dunes (Fortsetzung) 81

Ankündigung des »Pas d'armes de la Belle Pèlerine« 183, 187, 454

Anleitung zur Ausrufung des largesse-Dankes $60,132, \mathbf{2 8 3}, 288,290,378,400$, 455

- zur Blasonierung heraldischer Farben in Tugenden und Edelsteinen 68, 76, 158

- zur Darstellung von Tieren in den Wappen $138,332,456$

- zur Durchführung einer Legation durch die Herolde 45, 378

- zur Verkündung von Turnieren und Tjosten (Prolog für die nachfolgenden Texte zur Verkündung von Turnieren) $124 \mathrm{f} ., 288,451$

Antoine de La Sale:

Jehan de Saintré 74, 279, 325, 328

Le traité des anciens et des nouveaux tournois $17,31,52,73,279,287,349$

Salade $74,315,319,328,360,370,373$

Arbre des batailles siehe Bouvet, Honoré

Argentaye-Traktat 44f., 90-92, $215 \mathrm{f}$.,

216f., 220-224, 242, 244f., 249f., 251, 254, 261, 327 f., 397, 407, 456

Aristoteles: Dits 118

Metaphysik 240f.

Armagnac, Jacques d': Turniertraktat 73, 418

Aufzählung der christlichen Königreiche 76

- der drei Ordnungen, der höchsten kirchlichen Würdenträger, der sechs Weltalter, der sieben Menschenalter etc. 115, 143 , $152,158,460$

- der französischen Könige von Ludwig VIII. bis Karl VII. 170, 334, 461

- der Fürsten und der Bischofsstädte Frankreichs 71, 76, 88f., 121, 146, 150, 170 f., 173, 183, 185f., 192, 202, 226,
331 f., 378-394, 395, 397 f., 401 f., 405, 413-415, 433, 439, 459

- der Fürsten und der Bischofsstädte Frankreichs - blasoniert $389 f$.

- der geistlichen und weltlichen Hierarchie $183,185,378,460$

- der Kurfürsten und Erläuterung der drei Kronen des Kaisers (Latein) 158

- der Neun Helden 76, 78, $170 \mathrm{f} ., 217 \mathrm{f}$., 332,460

- der Neun Helden in Versen 460

- der Neun Heldinnen 216f., 332, 461

- der savoyischen Herrschaften 183 , 185 f., 395, 460

- der Städte Philipps des Guten 143, 146, 149f., 395, 459

- der Tugenden des Adels 183, 332, 460

- der Verwandten und Freunde des Herzogs von Savoyen 183, 186, 332, 460

- der vier Nationen der Christenheit $170 \mathrm{f}$., 332, 459

- verschiedener heraldischer Figuren 138,457

- von Tugenden, Sünden, Lebensalter, Weltenalter etc. (»Aucunes enseignemens de la foi catholique «) 115

- zum katholischen Glauben (»Table de la foi catholique «) 144, 151

Aurispa, Giovanni: Débat de honneur (übers. v. Jean Miélot) 80, 143, 150, $333,360,425$

Ballard, William (Wappenkönig March): Bericht zu einem Turnier anläßlich der Hochzeit des Herzogs von York (1478) 40

Bericht über den gerichtlichen Zweikampf zwischen Gérard de Mortagne und Seigneur de Chin et Busignies (Nancy, 1386) 197

Bericht zum »Pas d'armes de l'Arbre d'or« 77

Bericht zur Überführung und den Obse- 
quien Herzog Philipps des Guten und der Isabella von York (1473) 77

Bernhard von Clairvaux:

Brief an den Ritter Raimond du Châtel-

Ambroise 116

Kurzer Text zur Heiligen Jungfrau Maria 116

Beschreibung einer türkischen Gesandtschaft 1461 in Saint-Omer 158

Blason de toutes armes et escutz 344-346

Blason des couleurs 343-347

Blasonierte Aufzählung der vier Fürsten der Christenheit 71, 216, 458

Blasonierungstraktat in Form eines Dialoges $69, \mathbf{7 1}, 75 \mathrm{f} ., 78 \mathrm{f}$., 90-92, 106, $111 \mathrm{f} ., 120 \mathrm{f} ., 124,128,137,143,145$, $170 \mathrm{f} ., 216,221,247 \mathrm{f} ., 331 \mathrm{f} ., 334 \mathrm{f}$., 336-350, 354f., 357f., 366, 397, 401, 407, 412, 425, 428, $431 \mathrm{f} ., 439,455$

Blasonierungstraktat mit einer sich anschließenden Wappensammlung 158

Bluemantle (Persevant): Bericht über Ereignisse am Hof Eduards IV. (1471/72) 41, 196

Boccaccio, Giovanni: Dekameron 156

Bouvet, Honoré: L'Arbre des batailles 75f., 90, 92, 102, 106, 113f., 117-119, 124, 204, 208, 210-214, 216, 221, 340, 343, 351-355, 358f., 361, 372, 399, 412, $424,440,463$

Auszug zu den gages de bataille 63,75 , 79, 88, 124, 130, 132, 286, 327, 397, 450 Auszug zu den Wappen 75, 79, 88, 124, 457

Brant, Sebastian: Das Narrenschiff 27

Bretonische Wappenrolle 216f., 223, 334, 458

Brief des Wappenkönigs Calabre 80, 88, 106-108, 170, 172, 174f., 227, 253, 257, 265-271, 274-276, 396, 410f., 444

Brief zur Wahl eines Wappenkönigs 170, 172, 174, 175, 239, 394, 445

Briefsteller 170, 172, 174, 175, 216f., 332, 378, 397, 439, 463

Brüninghausen, Hermann von (Wappenkönig der Ruyers): Wappenbuch des Hubertusordens 43

Burgundisches Formular für einen gage de bataille 121, 122, 143, 146, 169, 208, 240, 285f., 309, 396, 440, 449

Chandos (Herold): Reimchronik $38 \mathrm{f}$. Charolais (Herold): Bericht über das Or- densfest des Ordens vom Goldenen Vlies in Valenciennes (1473) 40, 77

Chartier, Alain: Bréviaire des nobles 73, 76

Le Lai de Paix 216f., 335, 465

Lounge de Paris 216f., 465

Chastellain, Georges:

Epistel an Herzog Philipp den Guten von Burgund (1456) 143, 151, 335, 416

Hector et Achille 156

Chaucer, Geoffrey: House of Fame 35

Chestre (Herold):

Bericht zu den Obsequien Richards, Her-

zog von York 40

Bericht zum »Pas du perron fée« 40

Bericht zur »Emprise de la Fleur de

Souvenance 40

Epitaph Richards, Herzog von York 41

Christine de Pizan:

Einseignement moraux 116

Livre des faits d'armes et de chevalerie (überarbeitete Fassung) 117, 119

Chronik der französischen Könige bis Karl VII. 96, 100, 138 f., 142 f., 146f., 150f., 334f., 461

- zur Geschichte Frankreichs zwischen 1400 und 146777

Chroniken zur Geschichte der Stadt Metz 116

Chronique du Religieux de Saint-Denys (Michel Pintoin) 37

Chroniques de Bretagne 93, 122

Claas Heynenzoon (Herold Gelre, dann Beyeren): Ehrenreden 39

Geschichte Hollands 39

Wappenbuch 39, 42, 181

Weltchronik 39

Conrad Kyeser: Bellifortis 84

Corpus Iuris Civilis: Digesten $241 \mathrm{f}$., 244, 249f., 251, 254

Courtois, Jean (Herold Sicile):

Kurzer Exkurs zu adeligen Zweikämpfen 130, 323, 454

Kurzer Exkurs zum gage de vilains 130, 132, 136, 327, 329f., 396, 404, 428 f., 450

Prolog zum »Kompendium des Herolds Sicile« 57-66, 71, 85, 94, 129, $132 \mathrm{f}$., 136, $344 \mathrm{f} ., 414$

Zur Durchführung eines Tournois voluntaires 130-132, 288, 290, 451

Zur Vorgeschichte des Heroldsamtes $65,130,136,444$ 
Créquier-Traktat 45, 86f., 170 f., 172 f., 457

Croissant (Persevant): Gedicht auf das Wappen des René d'Anjou 179

Débat des hérauts d'armes de France et d'Angleterre 37, 392, 394

Des Teufels Netz 34

Dits sechs sorgenvoller Magister in Paris $121, \mathbf{1 2 3}$

Doctrinal sauvauge 116

Dokumente zur Geschichte der Stadt Metz 116

Du Breuil, Guillaume: Stilus curie Parlamenti 302, 304-308

Empereur-Traktat 74, 76f., 79, 89f., 99, 111-114, 121 f., 124, 126-128, 132, 143 f., 170f., 173, 182f., 185, 188, 192, 201, 211, 213, 216f., 222, 283, 328, 331, 333, 360-378, 385, 389, 397 f., $401 \mathrm{f}$., 405, 413-415, 417, 433, 439, 462

Enseignement de la vraye noblesse siehe Hugues de Lannoy

Enseignements paternels siehe Hugues de Lannoy

Epiloge und excuse des »Kompendiums des Ludwig von Brügge« $65,86,94,191-$ 193, 195, 200, 202 f., 299

Epistel über die gerechte Herrschaft 120, 123, 143, 145, 333, 397, 466

- über die Nichtigkeit des menschlichen Daseins 78, 120 f., 123, 143, 145, 154, 333, 397, 440, 467

Epitaph des Jacques d'Esch $\mathbf{1 1 8}$

- Herzog Philipps des Guten von Burgund 78

Escouchy, Mathieu d': Chronique 15

Faits des Romains 241, 249, 251, 254, 261

Farbentraktat $45,68,71,77 \mathrm{f}$., 90, $105 \mathrm{f}$., 109, 111 f., 143, 146, 332, 345, 350-360, $399,405,412,415,439,457$

Faucquet, Jean: Textsammlung (Briefe, Wappengedichte etc.) 143, 158-162

Fondement et origine des tiltres de Noblesse $\&$ excellens estatz de tous Nobles ... 164

Friedrich I., König von Aragon: Wappentraktat 70

Froissart, Jean: Chroniques 32, 36
Gaguin, Robert siehe Julius Caesar: De Bello Gallico (frz. Übersetzung)

Gardé, Fabien (Herold Valois): Memoiren 13

Garter (Wappenkönig): Bericht zum »Pas d'armes de l'Arbre d'or « 40

Gedicht auf das Wappen des René d'Anjou 178

Gedichte über die Einnahme Luxemburgs 78

Geleitbrief für den Persevanten Jamais (1415, Karl VI., König von Frankreich) $16,18,30,366$

- für den Herold Missenland (Friedrich IV., Markgraf von Meißen) 16, $26 \mathrm{f}$.

- für den Persevanten Regnaut Talmas (Jean, Seigneur von Rouverel) 24

- für den Wappenkönig von Brabant (1420, Johann IV., Herzog von Brabant) 16,18

Genealogie der französischen Könige von Ludwig VIII. bis Karl VII. (1429) 140, 170, 174f., 178, 334, 461

- der Grafen von Boulogne 138-141, 334, 461

- der Grafen von Holland und Zeeland 138, 140 f., 334

- Peters I. von Luxemburg und der Marguerite des Baux (1470) 43, 138, $140 \mathrm{f}$., 334,462

Geoffrey von Monmouth: Historia regum Britanniae 156

Goldene Bulle von 1356 368-371, 374, 463

in französischer Übersetzung 111-114, 333

Grandes Chroniques de France 156

Grünenberg, Konrad: Wappenbuch 70, 221

Hans Judensint: Lied zu Peter von Hagenbach 34

Hérard, Jehan: Heroldstraktat $63,88,132$, 135, 215 f., 219, 238, 253, 274, 396, 440, 443

Heroldsschwur 79, 100, 105, $120 \mathrm{f} ., 143$, 145, 183, 185, 192, 201, 204, 238, 240, $412,439,444$

Heroldstugenden und allgemeine Anweisungen zum Amt 96, 143, 145, 239, 443

Heroldsvereinigung von Saint-Antoine-lePetit-Dokumente zur Gründung 19f., 
$22,27,86,108,130,132,135,227,239$, $273,394,410,447$

Hosenbandorden: Statuten 123

Instruction d'ung petit prince siehe Lannoy, Hugues de

Interpretation des Wortes »herault « und kurze Ausführungen zu den Engeln als Vorläufer der Herolde 158

Inventar der königlichen Bibliothek zu Blois 205

Isaac, Thomas (Wappenkönig Toison d'or): Heroldstraktat $183 \mathrm{f}$.

Isidor von Sevilla: Etymologiae 246, 251

Jacobus de Cessolis: Échecs moralisés (frz. Übersetzung) 79f., 88f., 92f., 111, 136, 144, $249 \mathrm{f}$.

Jacques de Longuyon: Voeux du paon 221, 243

Jacquet de La Ruelle (Persevant Talent): Gedicht über die Schlacht von Othée (1408) 35

Jan Dyllen: Klage der Tugenden 25

Jean Le Court, dit Brisebare: Restor du Paon 25

Jean Miélot siehe Aurispa, Giovanni: Débat de honneur; Montemagno, Buonaccorso da: Controversie de noblesse

Jehannot, Etienne: Instruction de chevalerie et exercice de guerre 117, 119

Johann Holland (Herold): Spruchdichtung auf das Schaffhausener Turnier 1392 250

John Wrythe (Wappenkönig Garter): Garter Book 42

Julius Caesar: De Bello Gallico (frz. Übersetzung von Robert Gaguin) $166 \mathrm{f}$.

Juvenal des Ursins, Jean: Brief an die französischen Generalstände in Blois (1433) 424

Kapitel des »Pas d'armes de l'arbre Charlemagne « (1443) 177, 454

- einer emprise des Philipp von Ternant (1446) 183, 187, 455

- einer emprise eines François von Ravorée $183, \mathbf{1 8 6}, 454$

- einer emprise vierer savoyischer Ritter 183, 186f., 455

- einer Tjost, veranstaltet von drei Schildknappen aus der Familie de Villette 183, 186, 188, 453
Karthagobrief 62f., 105,106f., 108f., 111, 113, $130 \mathrm{f} ., 132,135 \mathrm{f} ., 183,185,238$, 240, 246f., 250, 253, 257, 337-339, 346, $358,394,400,407,412,415,425,432$, $439,442 \mathrm{f}$.

Konkordanztabelle Jahreskosten $\quad$ 144, 168

Kurze Anweisungen zur Durchführung von Obsequien 158

- Chronik Frankreichs zwischen 14031442 143, 146, 148, 150, 461

- Sammlung der wichtigsten Wappen siehe Wappensammlung der wichtigsten Wappen

Kurzer Exkurs zu adeligen Zweikämpfen siehe Courtois, Jean (Herold Sicile)

- Exkurs zum gage de vilains siehe Courtois, Jean (Herold Sicile)

- religiöser Text zu Adel und Ehre 76

- Text zur Heiligen Jungfrau Maria 116, 416

L'art de chevalerie selon Végèce siehe Christine de Pizan: Livre des faits d'armes et de chevalerie

La Jaille, Hardouin de: Formulaire des gaiges de bataille 322,328

La Marche, Olivier de: Epistre pour tenir et celebrer la noble feste du Thoison d'Or 17

Estat de la maison du duc Charles de Bourgoigne, dit le Hardy 28, 77, 360 Le livre de l'advis de gaige de bataille (1494) 284, 309, $321 \mathrm{f} ., 328$

Mémoires 28, 177, 187, 324

Traicté d'un tournoy tenu à Gand par Claude de Vauldray, seigneur de l'Aigle, l'an 1469 (v.st.) 77, 287, 324

La nouvelle maniere de blasonner les couleurs en armoirie et metaulx 344

Lannoy, Ghillebert de: Instruction d'ung petit prince siehe Lannoy, Hugues de

Lannoy, Hugues de:

Enseignements paternels 426, 428

Enseignement de la vraye noblesse 426

Instruction d'ung petit prince 426, 432

Le Baud, Pierre: Genealogie der Könige, Herzöge und Fürsten der Bretagne (1486) 216-218, 334, 462

Le Bouvier, Gilles (Herold Berry): Armorial de France 19, 42, $273 \mathrm{f}$.

Les chroniques du roy Charles VII 39

Livre de la description des pays 39, 379f., 389-394 
Recrouvement de Normandie 39

Le Féron, Jean: De la primitive institution des hérauldz et poursuivans d'armes 164

Le Forestier, Jean (Herold Bourgogne): Bericht über eine Mission nach Cambrai (1534) 22

Le Franc, Engherant (Herold von Valenciennes): Ditiers faits et armoriés 43

Le Tur, Guillaume: Traktat über die Gerichtsbarkeit des Konnetabel 88, $170 \mathrm{f}$., 176, 333, 397, 413, 463

Lefèvre, Raoul: Recueil des Histoires de Troyes 156

Lefèvre de Saint-Rémy, Jean (Wappenkönig Toison d'or):

Bericht zu den Taten des Jacques de Lalaing 39

Chronique 39

Traktat zu den Wappenminderungen 39, $48,67,86,138,143,147,158,298,332$, $397,440,456$

Limbourg (Herold): Bericht zum »Pas du perron fée « 40

Livre d'honneur 75

Livre de la Table de Pruce 75

Livres des armes - Blasonierungstraktat 90-92, 105, 106, 337, 357, 456

Livro de Arautos (Länderbeschreibung eines portugisieschen Herolds, Latein) 42,252

Lobpreis auf die Stadt Paris 217f., 335, 456

Luxembourg (Herold): Blason de Lalaing 42,359

Mailles, Jacques de: Histoire du Chevalier Bayard 30

Mariengebete 115

Martorell, Joanot: Tirant lo Blanc 31

Matthaeus Parisiensis: Chronica maiora 370

Merlin de Cordebeuf: Projekt für ein Turnier nach der Mode der Zeit des König Artus 49, 179, 291, 403, 418f., 436, 453

Mézières, Philippe de: Songe du vergier 157

Auszug zu den gages de bataille 79, 88, 124,450

Auszug zu den Wappen 79, 88, 124, 457

Molinet, Jean: Chroniques 32, $159 \mathrm{f}$.
Monstrelet, Enguerran de: Chronique 24, 32, 36, 177

Montemagno, Buonaccorso da: Controversie de noblesse (übers. v. Jean Miélot) 143, 149f., 151, 333, 425, 464

Montjoye (Wappenkönig): Bericht zum Turnier anläßlich des Einzuges der Königsbraut Maria von England 1514 in Paris 40

Montjoye-Traktat 24, 49, 78, 102, $137 \mathrm{f}$., 143, 145, 165, 183-186, 190, 204f., 208, 237f., 240, 253, 262-282, 329, 400, 402, 406, 411, 440, 442

Navarra (Herold): Armorial de France 42

Obsequien des Bertrand du Guesclin 76, $170,284,448$

- des Gérard de Mortagne (†1391) 192, 196f., 284, 448

- des Jakobs II. von Luxemburg $(\dagger 1517)$ 299

- des Jean IV. du Bois, Seigneur von Tenques $(\dagger 1510) \quad 155$

- des Louis de Male, Grafen von Flandern 197

- Peters II. von Luxemburg (†1482) 105, 110, 284, 448

Obsequientraktat $48,66,77,79,89,100$, 105, 110, $120 \mathrm{f} ., 129,132 \mathrm{f} ., 143,145$, $155,183,185,188,192,195,198,202$, 283, 291, 292-300, 396, 402f., 408, 412, $428,434,439,447 \mathrm{f}$.

Ordene de chevalerie 115, 283

Ordonnanz des Johann von Bourbon, Konnetabel von Frankreich, zur Immunität der französischen Herolde 20, 278

- von 1495 zu den Herolden in Burgund 20

Ordonnanzen des Thomas of Woodstock zu den Zweikämpfen $120 \mathrm{f}$., 130, $143 \mathrm{f}$., 169, 192, 208, 213, 285f., 323, 396, 413, 439,449

- zu den gages de bataille in Frankreich $52,74,76,79,124,130-132,143$, $170 \mathrm{f} ., 183,185,192,208,281,285 \mathrm{f}$., $291 \mathrm{f} ., 300-331,360,374,396,398$, 402-404, 413, 415, 417, 431, 433, 439, 449

- zu den Obsequien des Königs 60 f., 283, 448

Orléans (Herold): Bericht zum Turnier von Sandricourt 40 
Patenôtre des gens d'armes 117

Perceforest 25, 35

Perreau, Louis de: Discours de l'origine, office, charge, progrès, privileges et immunitez des roys et herauts d'armes de France (1533) 23

Philipp von Novara: Les quatres âges de l'homme 116

Philippe de Beaumanoir: Coutumes de Beauvaisis 307

Piat, Jean (Herold Genève): Testament 14, 23

Piccolomini, Aeneas Silvius (Pius II.): De origine heraldorum $\mathbf{8 0}$ f., 221, 237, 253

Pierre Choque (Herold Bretagne): Bericht über die Obsequien zum Tod der Anne de Bretagne 41 Berichte über die Hochzeitsfahrt und -feier der Anne de Foix (1502) 41 Übersetzung des Gedichtes von Guillaume Brie zum Untergang der »Cordelière« 43

Prinsault-Traktat 69-71, 73, 78, 90-92, $191 \mathrm{f} ., 200,204,207,213,216,219,221$, 223, 331, 349f., 357, 397, 432, 456

Privilegien der Herolde (allgemein) 52,90, 105, 112, 132, 135, 238, 445

Privilegien der Herolde auf Turnieren 111 f., 202, 238, 396, 445

Prolog des »Kompendiums des Ludwig von Brügge« 94, 128, 191-193, 195, 198200, 203,

- innerhalb des »Angevinischen Heroldskompenidums « 95

Quiéret, Gauvain:

Complainte de Grèce 157

Guidon 157

Traitté de la ressource de France 157

Trialogue 156f., 203, 299, 429

Vision et traité de la vie humaine 157

Raimundus Lullus: Libre del ordre de cavayleria 423,426

Rechte der französischen Marschälle 208, 463

Reimchronik zu Peter von Hagenbach 34

Renart, Jean: Roman de la Rose ou de Guillaume de Dole 33

René d'Anjou: Livre des tournois 73, 418, 436

Revel, Guillaume de (Herold Auvergne):
L'armorial d'Auvergne, Bourbonois et Forestz 20, 42, 278

Richental, Ulrich: Chronik des Konstanzer Konzils (1414-1418) 36

Roman de la Rose 157

Ryneck, André de: Wappenbuch 70, 221

Selon les dits-Traktat 24, 79f., 88, 90, 92f., 99, 105-109, 111, 114, 120-122, 128, 130, 132, $135 \mathrm{f} ., 141,143 \mathrm{f} ., 183$, 185f., 192, 201, 208, 215f., 220, 222 , 237-239, 240-262, 270, 275, 280-282, 338, 396, 405, $411 \mathrm{f} ., 425,439,441 \mathrm{f}$.

Suppliken:

Erste Supplik (Comme il vous appertiegne; 1408 [?]) 18-20, 27, 48, 59, 63, 79, 86, 100, 105f., 108, 111, 113, $120 \mathrm{f}$,, $132,135,143,145,170,172,183,185$, 227, 237 f., 252, 259f., 274, 394, 396, $411 \mathrm{f} ., 425,439,445 \mathrm{f}$.

Zweite Supplik (Comme d'ancienneté) 18f., 48, 59f., 63, 79, 100, 105, 111, 113, 120f., 132, 135, 143, 145, 170, 172, 237f., 259f., 394, 396, 412, 425, 439, 456

Dritte Supplik (Arras, 1435 [n.St.]) 19, 59f., 63, 86, 101, 130, 132, 135 f., 170, 227, 260, 274, 394, 396, 425, 456f.

Vierte Supplik (Comme il soit ainsi) 20, 112, 170 f., 227, 238 f., 260 f., 268, 273 f., 394, 396, 457

Supplik des Wappenkönigs Garter an den englischen König $24 f$.

Taillevent (Michault le Caron): Songe de Thoison d'or 78

Titulatur König Heinrichs von Kastilien 96f., 170, 459

Titus Livius: Les gestes romains (frz., angeblich von Robert Gaguin angefertigte Übersetzung der dritten Dekade des $» \mathrm{Ab}$ urbe condita $\ll$ ) 95, 143, 164-168, 230, 417

Tjost der Belle Maurienne (1454) Ausführliche Ankündigung 170, 453 Kurzform der Ankündigung 170, 453 Tjostankündigung vierer Ritter 170, 453

Tournai, Jean de (Wappenkönig Bonnes Nouvelles): Bericht über die Taufe Emmanuel-Philiberts von Savoyen (1528) 41

Traktat über den Ursprung der Pelzwerke in den Wappen 87, 170, 332, 397, 458 
- über die Ernennung eines neuen Persevanten, Herolds etc. 132, 135, $137 \mathrm{f}$., 141, 239, 439, $444 \mathrm{f}$.

- über die fünf militärischen Ämter 45, 72, 143, 145, 171, 443

- über die Kleidung der Krieger zu Pferd und zu Fuß 179

- über die Pilgerschaft 115

- über die richtige Ernährung von Kleinkindern 78

- über die Ritterschaft 65, 130, 132, 191, 283, 333, 347, 425-428, 432, 464

- zu den französischen Wappenprovinzen 53, 279

- zur Durchführung eines gage de bataille 216, 450

- zur Krönung des französischen Königs in Reims 71, 158

Turnierankündigung für Dijon nach Ostern 143, 152 f.

- Weiß gegen Rot 143, 152 f.

Über die Stadt Rom und ihre Kirchen (Latein) 142

Unterweisungen des Cato an seinen Sohn 116

Valera, Diego de:

Preheminencias y cargos de los oficiales d'armas 51

Traité de noblesse (übers. v. Hugo de Salve) 96, 138, 150, $191 \mathrm{f} ., 198,200$, 207, 209, 333, 425, 464

Verhaltensregeln zum Kirchenbesuch 116, 416

- zum Tagesablauf 116, 416

Verlorenes Heroldskompendium von Nicolas Villart (Herold Calabre) u. a. $86 \mathrm{f} .(?), 227,269,410 \mathrm{f}$.

Villart, Nicolas (Herold Calabre): Brief über das Amt der Herolde siehe Brief des Calabre

Villiers, Jean de: Le livre pour gaige de bataille 300, 309-314, 317-319, 328f.

Wappenbuch Gorrevod 360

Wappensammlung (didaktisch) 78

- der wichtigsten Wappen 170-172, 176, 217, 334, 413, 458

- mit den wichtigsten königlichen und fürstlichen Wappen 76
- Vergier de France 216, 458

- zu Brabant, Böhmen, Deutschland, Beauvaisis und weiteren französischen Wappenprovinzen sowie den christlichen Königen, den Sarazenen etc. 76

- zu den christlichen Königen 71, 216, 458

- zu den zwölf Pairs von Frankreich 216, 458

- zu Herzögen und Grafen 71

- zum Königreich Aragon 76

- (weitere) 71, 158f.

Wavrin, Jean de: Recueil des Croniques et Anchiennes Istories de la Grant Bretaigne 32

Weitere Form der Ankündigung einer Tjost 138f., 453

Wie Turniere à présent auszurufen sind (Turniere, Tjosten, Verbote) 124f., 130, 132, 135, 183, 288-290, 312 f., 439, 452

Wiener Kompilation 78f., $120 \mathrm{f}$., 124, 126-130, 185, 221, 285, 288, 290, 307310, 312-314, 317f., 326, 329, 367, 369 , 376,418

$\mathrm{Zu}$ den Aufgaben der Herolde auf einem Turnier à présent $52,63,79,89,124 \mathrm{f}$., 130, 132, 183, 288, 290, 439, 450f.

$\mathrm{Zu}$ den Persevanten und deren Ausbildung 105, 400, 443

Zum Anbringen der Banner auf den Turnieren $76,143,152,451$

Zur Ankündigung der Turniere nach der alten Manier 132, 135, 153, 288f., 452

- der Turniere zu Zeiten des König Artus 79, 96, 124, 132, 135, 143, 145, 288290, 418, 440, 452

Zur Durchführung der Turniere nach der alten Manier 76, 130, 132, 135, 153, 288f., 418, 451

- eines Tournois voluntaires siehe Courtois, Jean (Herold Sicile)

- von Turnieren und Buhurten 89, 112, $125,192,239,288,445,451$

Zur Rüstung der Turnierenden in den alten Zeiten 76, 130, 132, 135, 289-291, 418, 451

Zur Vorgeschichte des Heroldsamtes siehe Courtois, Jean (Herold Sicile)

Zusammenfassung des Turniers von Compiègne $129 \mathrm{f} ., 132,135,288 \mathrm{f}$., 454 